\title{
Les suffixes diminutifs dans la toponymie romane des Pyrénées *
}

\section{Louis BALMAYER **}

Nous avons entrepris il y a quelques années l'étude systématique des noms de lieux dérivés en ibéro-roman, ce qui représente un champ d'étude assez vaste pour être divisé en plusieurs sections. La première, sur le point d'être terminée, portait sur les suffixes romans proprement locatifs et parmi les plus employés. Une seconde partie, à peine commencée, sera consacrée à une autre catégorie de suffixes, très employés eux aussi pour la formation des noms de lieux; les suffixes diminutifs. Au point de vue géographique ces recherches portent sur le domaine ibéro-roman, celui où les dialectes issus du latin ont confondu $b$ faible et $b$ fort, autrement dit ne connaissent pas la fricative sonore $v$, et ceci à quelques exceptions près comme le portugais central et méridional ou le baléare et le valencien non «apitxat». Ces dialectes comprennent, on le sait, outre ceux de la Péninsule Ibérique, le gascon et le languedocien parlés au nord des Pyrénées. C'est donc cette chaîne qui forme le centre de cette partie de la Romania, non seulement par sa position dans l'espace mais aussi au point de vue linguistique, étant donné que le trait phonétique distinctif énoncé plus haut est attribué au substrat basque qui est à l'origine de la particularisation de ces parlers par rapport aux autres langues romanes. Cette importance se reflète dans la répartition et l'emploi des suffixes toponymiques.

M. GuIter à montré dans son article «Onomàstica del Pireneu mediterrani» paru dans «Miscellanea Barcinonensia», $\mathbf{n}^{\circ} 21$, que la zone cantabro-pyrénéenne marquait une prédilection très nette pour les suffixes en -etu et -anu, avec prédominance particulière du second dans l'isthme. Dan notre communication au $\ll V^{\mathrm{e}}$ congrés international $d^{\prime}$ études linguistiques méditerranéennes» de Málaga, en 1973, intitulée «La répartition des dérivés romans dans la toponymie de la Peninsule Ibérique», sur des

* Comunicación leída en las Sesiones de la VI Sección: Filología, Lingüística y Literatura pirenaicas, en Seo de Urgel, 1974.

** Prof. Chargé de Cours. Faculté de Lettres et Sciences Humaines. Université Paul Valéry. Montpellier. 
bases semblables et avec des méthodes statistiques différentes nous arrivions au même résultat. Enfin, dans un travail sur «les dérivés toponymiques en -anu et -acu dans les Pyrénées», présenté au «XIe congrès international des sciences onomastiques» tenu à Sofia en 1972, nous avions pu montrer que le suffixe - $a c u$ n'atteignait la frontière politique avec une densité supérieure à celle de -anu que dans les cantons d'Oloron et Argelès, et, d'une façon moins nette, dans le département de l'Ariège. L'isthme des Pyrénées apparaît au point de vue linguistique, et donc ethnique, comme une unité territoriale et, à une époque récente seulement, comme une barrière. Ceci expliqué que nous ayons eu la curiosité de commencer l'étude des noms de lieux diminutifs par cette région, malgré les inconvénients que cela peut comporter.

Nous avons recensé, en nous restreignant à la toyonymie majeure et en prenant pour base le «Nomenclátor de las ciudades, villas, lugares, etcétera», de 1960 d'une part, et, de l'autre, la «Nomenclature des communes» des imprimeries Oberthur, de 1965, les dérivés en -ellu, -eolu/-iola/ -olu, -icellu, -culu, -inu, -ittu, -iccu, -occu, -ete ${ }^{1}$. Chaque fois que nous l'avons pu nous nous sommes référé aux formes anciennes ou aux étymologies fournies par le «Diccionari català, valencià, balear» d'ALco ver et Moli, la «Toponimia aragonesa medieval» d'Agustín UBieto, «Catalunya Carolingia d'Abadal», le «Dictionnaire des noms de Lieux de France» de Dauzat et Rostaing, etc. A partir de ces bases nous n'avons pas admis aveuglément tous les noms de lieux qui comportent un suffixe diminutif. Nous avons éliminé les noms qui font partie du langage vernaculaire et pour lesquels toute valeur diminutive s'est perdue. Le type en est les héritiers de castellu. En revanche nous avons admis un toponyme comme Vinyols (Tarragone) bien que le nom commun catalan vinyol désigne une jeune vigne, car dans ce sens il y a, au moins sous la forme de trace, une idée diminutive, et qu'il n'est pas exclu que le toponyme ait été formé pour désigner une petite vigne, de peu d'étendue. Nous avons rejeté encore les toponymes pour lesquels nous n'avons pas trouvé d'étymologie satisfaisante, même s'il s'agit, manifestement de formations diminutives. Enfin, nous n'avons pas tenu compte non plus des toponymes formés à partir $\mathrm{d}^{\prime}$ un nom de personne comme Bernadets (Hautes-Pyrénées) qui viendrait de l'anthroponyme germanique Bernard affecté du suffixe -ellu. Cependant, les toponymes comme Sabadell, Vendrell, en Catalogne, pour lesquels on peut admettre une autre étymologie (die sabbati, die veneris) ont été conservés.

Dans cet inventaire, des difficultés proviennent de la forme qu'ont pris les sufixes dans la langue moderne et qui, en l'absence de formes suffisamment anciennes peut provoquer des confusions: -ittu aboutit à -et en catalan, en languedocien, en gascon et parfois aussi en aragonais

1. Nous avons prérére exclure -one de cetae liste car il est tantôt diminutif tantôt augmentatif et semble prendre en toponymie d'autres valeurs encore. Il pourra à lui seul faire l'objet d'une étude à part. 

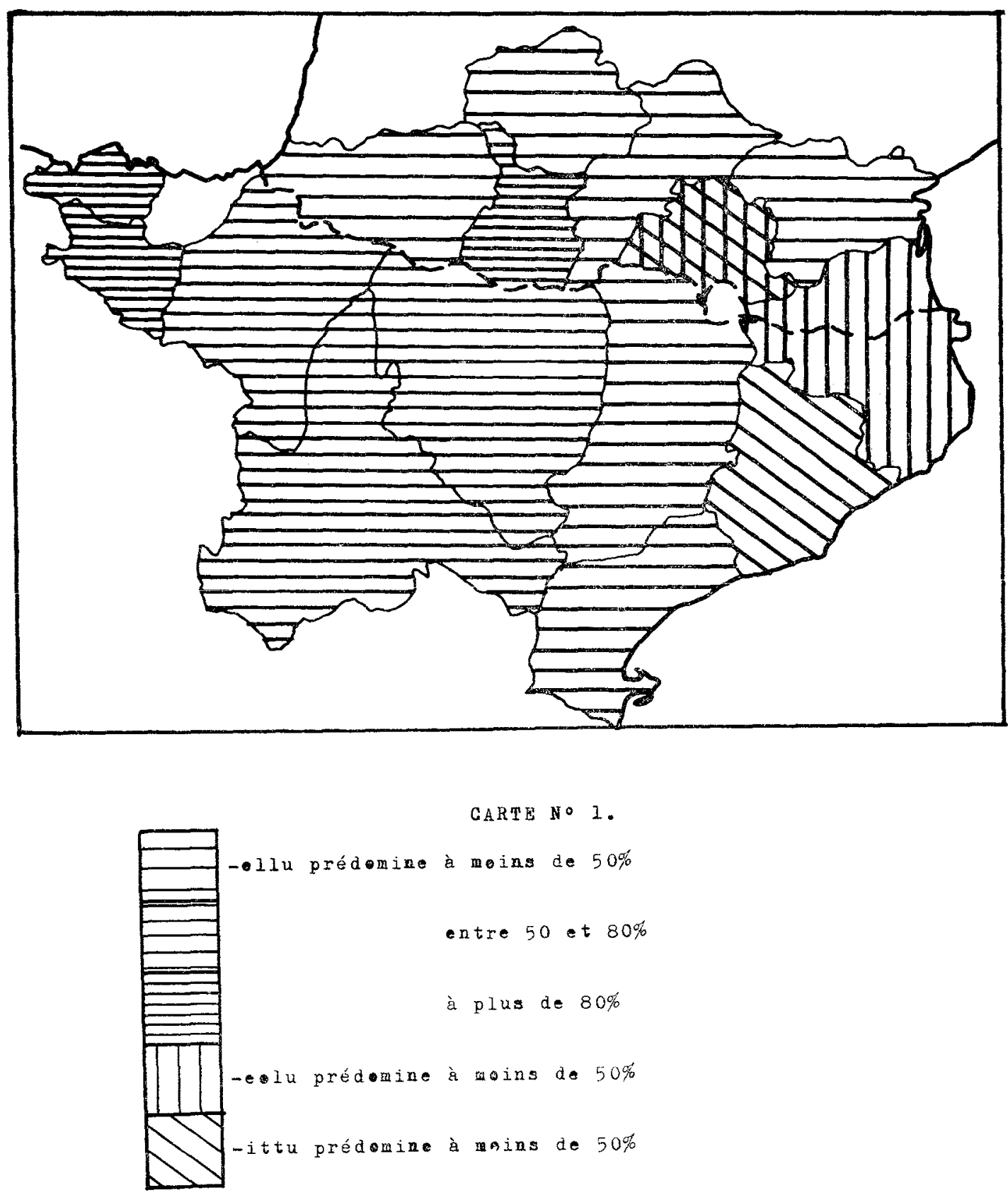

(Z. VICENTE dans «Dialectología espaôla», p. 297, recense les formes suivantes: -ete, -era, et, é). Il peut donc se confondre avec -etu. C'est ainsi que pour les deux localités de la province de Gérone du nom de Canet, malgré les formes suivantes citées par Balari dans «Orígenes históricos de Cataluña»: Canneto, 1017, Canet d'Adri; Canet, 1087, Canet de Verges, on peut envisager que l'on a à faire à l'étymon canna, = roseau, suivi de -etu (mais, en ce cas, $n$ double devrait aboutir à $n$ mouillé), on au pré-indoeuropéen *kan, = hauteur, suivi de -etu ou -ittu. Que le $n$ soit simple ou double, cela ne suffit pas à nous renseigner, car il peut s'agir d'une faute d'orthographe du scribe. Même s'il ne fait pas de doute que la base est un nom de végétal, il n'est pas exclu, bien que ce soìt là un critère que nous ayons utilisé, qu'on y ait adjoint le suffixe diminutif, ce qui s'est produit, sans doute possible apparemment, pour 
Rourell (Tarragone), par exemple. La topographie des lieux, dont nous n'avons, il est vrai, qu'une connaissance livresque, ne peut fournir, dans bien des cas, qu'une simple présomption: Selon MaDoz, le premier Canet est situé «en terreno llano, al pie de una montaña»; selon Carreras Candi ( $G$ Geografía de Catalunya»), le second se trouve en terrain montagneux et porte le même nom qu'un torrent. D'autres confusions possibles sont restreintes au domaine gascon. Dans ce dialecte le féminin -ella, ellas aboutit à -ère -ères et peut se confondre avec les héritiers de -aria, -arias; le masculin -ellu, -ellos, devenant -et, ets, prend la même forme que -ittu, -ittos. En catalan enfin, -iculu et -ellu aboutissent tous les deux à -ell. Pour tous ces cas, lorque les formes anciennes ne renseignent pas ou font défaut, la dernière ressource, bien fragile il est vrai, est le bon sens ou l'intuition!

Les résultats numériques sont les suivants:

1. NOMBRE DE TOPONYMES PAR PROVINCE OU DEPARTEMENT:

\begin{tabular}{|c|c|c|c|c|c|c|c|c|c|}
\hline & -ellu & - eolu & -icellu & - cuiu & $-i n u$ & $-i t: u$ & -otiu & Autres & Total \\
\hline Gérone $\quad . . . . . .$. & 18 & 22 & & 3 & & 15 & & & 58 \\
\hline Barcelone ..... & 32 & 24 & & & 1 & 33 & 3 & & 93 \\
\hline Tarragone ...... & 13 & 6 & & & 2 & 11 & 1 & & 33 \\
\hline Lérida......... & 23 & 17 & 2 & & 1 & 17 & & & 60 \\
\hline Huesca $\quad . . . . . .$. & 33 & 13 & 2 & & & 5 & & 2 & 55 \\
\hline Saragosse ....... & 18 & 8 & 1 & & & 2 & & 1 & 30 \\
\hline Navarre...... & 11 & 2 & & & & 1 & & & 14 \\
\hline Biscaye $\ldots \ldots \ldots$ & 7 & & & & & & & 1 & 8 \\
\hline Alava $\ldots \ldots \ldots$ & 6 & 1 & & & & & & & 7 \\
\hline Guipúzcoa ..... & & & & & & & & & $\mathbf{0}$ \\
\hline Aude ........... & 12 & 10 & 2 & 1 & & 11 & & & 36 \\
\hline Pyr.-Or. ........ & 2 & 4 & 2 & & & 2 & & & 10 \\
\hline Ariège $\quad . . . . . .$. & 2 & 5 & & 2 & & 5 & & & 14 \\
\hline Hte.-Gar. ...... & 14 & 3 & & 1 & & 11 & & 1 & 30 \\
\hline Htes.-Pyr. ..... & 16 & 2 & & & & & & & 18 \\
\hline Gers $\ldots \ldots \ldots \ldots$ & 8 & 3 & & & & 1 & & & 12 \\
\hline Bsses.-Pyr. .... & 7 & 3 & & & & 4 & & & 14 \\
\hline Total $\ldots \ldots$ & 222 & 123 & 9 & 7 & 4 & 118 & 4 & 5 & 492 \\
\hline
\end{tabular}

2. POURCENTAGES:

\begin{tabular}{|c|c|c|c|c|c|c|c|c|}
\hline Gérone ......... & 31 & 38 & & 5 & & 25 & & \\
\hline Barcelone.... & 34 & 26 & & & 1 & 35 & 3 & \\
\hline Tarragone ...... & 39 & 18 & & & 6 & 33 & 3 & \\
\hline Lérida .......... & 38 & 28 & 3 & & 2 & 28 & & \\
\hline Huesca .......... & 60 & 23 & 4 & 9 & & & & 4 \\
\hline Saragosse ....... & 60 & 26 & 3 & & & 7 & & 3 \\
\hline Navarre $\ldots \ldots .$. & 79 & 14 & & & & 7 & & \\
\hline Biscaye ......... & 88 & & & & & & & 13 \\
\hline Álava .......... & 85 & 14 & & & & & & \\
\hline Aude .......... & 33 & 28 & 6 & 3 & & 31 & & \\
\hline Pyr.-Or. ....... & 20 & 40 & 20 & & & 20 & & \\
\hline Ariège ......... & 14 & 36 & & 14 & & 36 & & \\
\hline Hte.-Gar. ...... & 47 & 10 & & 3 & & 40 & & \\
\hline Htes.-Pyx ..... & 89 & 11 & & & & & & \\
\hline Gers ............. & 67 & 25 & & & & 8 & & \\
\hline Bsses.-Pyr. .... & 50 & 21 & & & & 29 & & \\
\hline Ensemble ..... & 45 & 25 & 2 & 1 & 1 & 24 & 1 & \\
\hline
\end{tabular}


On observera que le suffixe le plus représenté est -ellu suivi par -eolu et -ittu qui sont à égalité entre eux (respectivement 45,25 et $24, \%$ ). Ceci est conforme à ce qu'écrit Bourciez dans "Les éléments de linguistique romane», sur la répartition des suffixes diminutifs dans les langues vernaculaires: -ellu et -eolu «restèrent productifs partout»; «c'est en Gaule et surtout en Italie que (-ittu) prit une grande extensión». Mais le suffixe -iccu qui, selon ZaMora Vicente ( Dialectología española»), «se viene considerando como típico del dialecto aragonés» est presque absent de la toponymie même dans cette région où, -écrit-il encore- -ellu «es abundantísimo sobre todo en la toponimia».

Si nous figurons maintenant sur une carte (núm. 1) le suffixe qui prédomine dans chaque province ou département, on voit apparaître trois zones: l'une, la plus étendue, pour -ellu, l'autre formée de la province de Gérone et des départements des Pyrénées-Orientales et de l'Ariège pour -eolu; la troisième enfin constituée par la seule province de Barcelone où -ittu l'emporte. En fait, telle répartition brutale des résultats chiffrés peut $n^{\prime}$ être pas le reflet exact d'une réalité. Il faut, pour pouvoir l'admettre comme telle, appliquer le test de Pearson, dit du $\mathrm{X}^{2}$, qui nous permettra de savoir si la répartition des dérivés entre deux régions est, ou non, aléatoire. Nous ferons ces opérations en apportant aux données les modifications suivantes: il ne ser tenu compte que des trois suffixes les plus représentés; -icellu sera compté avec -ellu car l'un n'est en fait qu'une variante de l'autre; il ne sera pas tenu compte des provinces de Biscaye et d'Álava pour lesquelles les données numériques sont insuffisantes et il faudra quelque circonspection pour interpréter les résultats dans la calcul des quels interviendront les données des Pyrénées-Orientales oú le nombre total des dérivés atteint à peine la dizaine. Done les $X^{2}$ de la distribution des dérivés en -ellu, -eolu et -ittu sont, dans la zone orientale de notre domaine, les suivants: Barcelone/Tarragone $=1,15$; Barcelone $/ \mathrm{Ge}-$ rone $=2,33 ;$ Barcelone $/$ Lérida $=1,13 ;$ Aude/Pyrénées-Orientales $=0,85^{2}$ Aude/Ariège $=2^{2}$; Ariège/Pyrénées-Orientales $=1,03^{2}$; Arigè $/$ Haute Garonne $=8,61^{2}$; Ariège/Lérida $=2,80^{2}$. A l'exception de celle qui concerne $\mathrm{I}^{\prime}$ Ariège et la Haute-Garonne toutes ces valeurs signifient qu'il y a entre 3 et 7 chances sur 10 pour qu'une répartition au hasard soit la même que celle qui existe effectivement (Cf. Ch. Mulder, «Initiation aux méthodes de la statistique linguistique»); autrement dit, malgré la première impression, il n'y a pas de différence significative dans le choix des suffixes diminutifs pour la toponymie entre ces provinces ou départements, et les trois zones que nous avions cru pouvoir distinguer n'existent pas. Cependant, le fait que trois de ces territoires sur quatre soient de langue catalane nous conduit à étudier la distribution de nos dérivés selon les aires dialectales et non plus en fonction des divisions administratives, souvent

2. Résultats peu significatifs car certains effectifs calculés sont inférieures à 5 . 
arbitraires. La répartition est alors la suivante: zone gasconne: BassesPyrénées, Hautes-Pyrénées, Gers, Haute-Garonne et Ariège gasconnes; zone languedocienne: Aude, Haute-Garonne et Ariège languedociennes; zone navarro-aragonaise: Navarre, Huesca, Saragosse; zone catalane: Tarragone, Barcelone, Gérone, Lérida, Pyrénées-Orientales; Pays Basque: Biscaye, Álava.

\begin{tabular}{|c|c|c|c|c|}
\hline & $-e l l u$ & $-e o l u$ & $-i t t u$ & Total \\
\hline Zone gasconne & $4: 4$ & 12 & 8 & 64 \\
\hline 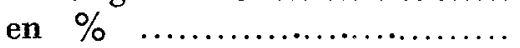 & 69 & 19 & 12 & \\
\hline Zone languedocienne ........... & 17 & 14 & 24 & 55 \\
\hline en $\% \quad \ldots \ldots \ldots \ldots \ldots \ldots \ldots \ldots \ldots \ldots \ldots \ldots$ & 31 & 25 & 44 & \\
\hline Zone navar.-arag. ................ & 65 & 23 & 8 & 96 \\
\hline 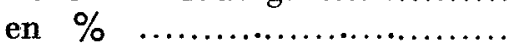 & 68 & 24 & 8 & \\
\hline Zone catalane ................... & 92 & 73 & 78 & 243 \\
\hline en $\% \quad \ldots \ldots \ldots \ldots \ldots \ldots \ldots \ldots \ldots \ldots \ldots \ldots$ & 38 & 30 & 32 & \\
\hline Pays basque ..................... & 13 & & 2 & 15 \\
\hline 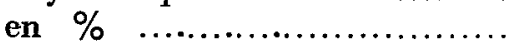 & 87 & & 13 & \\
\hline
\end{tabular}

On remarquera dès maintenant que -ellu l'emporte partout sauf, ce qui a peu de sens étant donné la faible portion de territoire représentée ici, mais paraît tout de même conforme à ce qu'écrit Bourciez, dans la zona languedocienne. $D^{\prime}$ autre part, dans la zona -ellu, le second suffixe le plus représenté est -eolu, excepté en Catalogne où c'est -ittu et où, par ailleurs, les trois suffixes offrent des pourcentages assez voisins. Le test de Pearson appliqué à ces zones donne les résultats suivants:

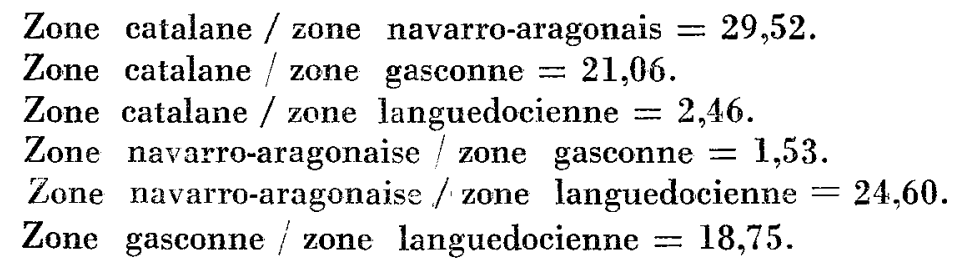

Ces valeurs révèlent une distribution non aléatoire sauf entre la zone navarro-aragonaise et la zone gasconne et entre la zone catalane et la zone languedocienne, mais il ne faut pas oublier que dans ce dernier cas l'échantillon languedocien est vraiment réduit, ce qui ne permet pas de tenir le résultat pour significatif.

Donc, la répartition des diminutifs toponymiques romans révèle, comme celle des autres suffixes que nous avons étudiés antérieurement, la remarquable unité linguistique, en ce domaine, de la zone pyrénéenne: partout c'est -ellu qui a été le plus employé suivi, ce qui est peut-être le plus intéressant par -eolu, sauf sur le territoire catalan où -ittu se trouve sensiblement à égalité avec le second. Cette unité qui se révèle ainsi dans la toponymie par-delà les différences dialectales, on peut raisonnablement l'attribuer à un même substrat linguistique pré-indoeuropéen qui ne peut être que le basque ancien, comme il apparaît nettement dans l'étude de 

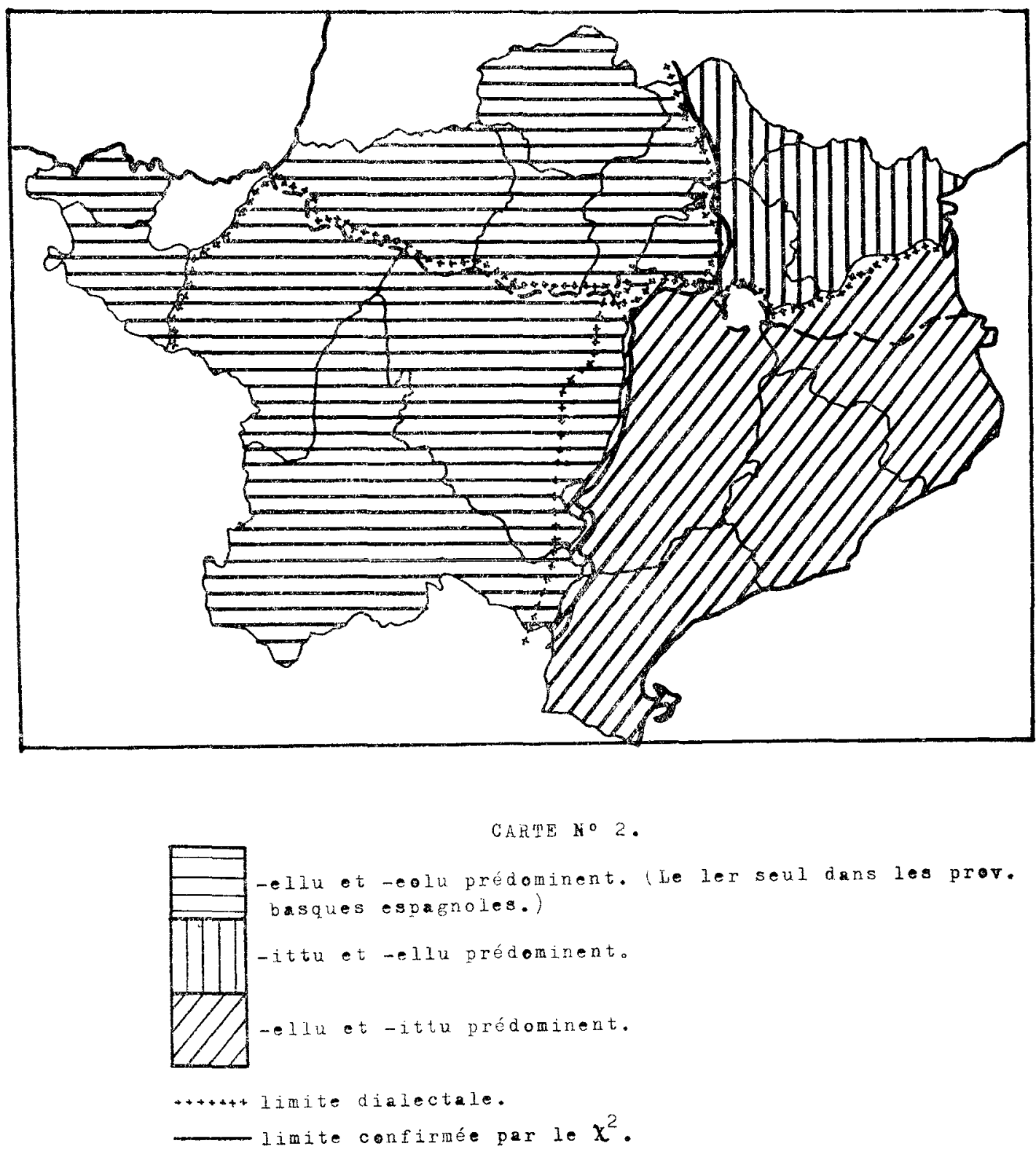

M. Guiter citée plus haut, oú les succès de -etu et -anu dans l'isthme est mis en relation avec la présence des suffixes basques -eta et -ain.

Bien sûr, ces conclusions seraient plus probantes si nous pouvions les replacer dans le contexte de l'ensemble de l'Ibéro-Romania. C'est là l'inconvénient auquel nous faisions allusion au début. Pour y pallier dans une certaine mesure nous avons fait des comptes estimatifs ailleurs: dans la province de Madrid, ellu l'emporterait à $80 \%$ pour $8 \%$ à -eolu et $7 \%$ à -culu; dans celle de Tolède on aurait -ellu, $61 \%$, -iculu, $16 \%$, -eolu, $11 \%$; dans l'Aveyron: -eolu, $43 \%$, ittu, $32 \%$, -ellu, $21 \%$; dans la Corrèze: -eolu, $50 \%$ et -ittu $20 \%$. 
